\title{
„Rechtsbewährung“ als Grundprinzip der Notwehr? Kriminologisch-empirische und verfassungsrechtliche Überlegungen zu einer Reformulierung von $\$ 32$ StGB
}

Johannes Kaspar"

A. Einführung

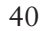

B. Die Funktionen des Prinzips der

„Rechtsbewährung“"

40

I. Notwehrerweiternde Funktion...... 42

II. Notwehrbeschränkende Funktion . . 42

1. Krasses Missverhältnis .......... 42

2. Provokation ................... 43

3. Enge persönliche Verbundenheit von Angreifer und Verteidiger... 44

4. Angriffe von schuldlos Handelnden

5. Notwehr gegen Schweigegelderpressung

C. Kritik am Prinzip der „Rechtsbewährung"

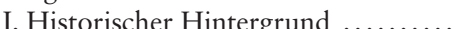

II. Zirkularität

III. Inhaltliche Unbestimmtheit

(1)

42

3

D. Rechtsbewährung als generalpräventive Komponente der Notwehr?...... 51

I. Hinweise für ein solches Verständnis von Rechtsbewährung ............ 52

II. Empirisches oder normatives Verständnis von Generalprävention?... 52

III. Verfassungsrechtliche Konsequenzen ........................... 56

E. Fazit: Restriktives Notwehrverständnis auf der Basis einer eingeschränkt-dualistischen Notwehrbegründung ....... 59

F. Zur Notwendigkeit einer Neurege-

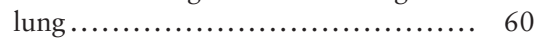

\section{A. Einführung}

Der Rechtfertigungsgrund der Notwehr ist bis heute Gegenstand einer lebhaften Diskussion im strafrechtlichen Schrifttum. Die Notwehrregelung wurde zwar bereits von Binding als besonders gelungen bezeichnet; ${ }^{1}$ und auch in der Begründung des Entwurfs von 1962 heißt es eingangs noch, dass sich das geltende Notwehrrecht „im allgemeinen bewährt" habe. ${ }^{2}$ Dennoch zeigen die vielen strittigen Fragen, die in Rechtsprechung und Lehre diskutiert werden, dass über Inhalt und Reichweite von \32 StGB keineswegs Einigkeit herrscht. ${ }^{3}$ Das gilt insbesondere im Hinblick auf die - für die Lösung konkreter Auslegungsprobleme wichtige - Frage der Grundprinzipien der Notwehr.

Hier stehen sich seit längerer Zeit drei Ansichten gegenüber. ${ }^{4}$ Nach einer Mindermeinung, die aber in jüngerer Zeit zunehmend mehr Gefolgschaft findet, ist die ratio

* Prof. Dr. Johannes Kaspar ist Inhaber eines Lehrstuhls für Strafrecht, Strafprozessrecht, Kriminologie und Sanktionenrecht an der Universität Augsburg. Der Beitrag ist Herrn Prof. Dr. Joachim Herrmann mit den besten Wünschen zum 80. Geburtstag gewidmet.

1 K. Binding, Handbuch des Strafrechts, Band 1, Leipzig 1885, 732 Fn. 5.

2 BT-Drucks. IV/7650, 156.

3 Vgl. nur T. Lenckner JZ 1973, 253.

4 Überblick bei C. Roxin, Strafrecht Allgemeiner Teil, Band I, 4. Aufl., München 2006, $\mathbb{1 5}$ Rn. 1 ff.; s. auch $P$. Kasiske Jura 2004, 833 sowie die umfassende Darstellung bei A. Engländer, Grund und Grenzen der Nothilfe, Tübingen 2008, 7 ff. 
von $\mathbb{S} 32$ StGB allein im Schutz individueller Güter zu finden. ${ }^{5}$ Die insbesondere von Schmidhäuser vertretene Gegenposition sieht die Notwehr demgegenüber allein im überindividuellen Aspekt der „Rechtsbewährung“ begründet. ${ }^{6}$ Die Notwehrhandlung, die sich gegen den rechtswidrig Angreifenden richtet, wird hier allein unter dem Blickwinkel der „Verteidigung der Rechtsordnung“ gesehen, während der damit zugleich bewirkte Schutz individueller Güter als Nebenwirkung und bloßer „Reflex“ der Verteidigungshandlung erscheint.

Gemeinsam ist den beiden bisher genannten Ansätzen, dass sie monistisch sind, d. h. von nur einem einzigen tragenden Grundprinzip der Notwehr ausgehen. Die herrschende dualistische Ansicht dagegen kombiniert beide Aspekte: ${ }^{7}$ Der Rechtfertigungsgrund des $\mathbb{S} 32$ StGB sei nur dann richtig erfasst, wenn man ihn auf Individualschutz und Rechtsbewährung stütze. Und tatsächlich sind Einwände gegen eine rein monistische Perspektive schnell bei der Hand. ${ }^{8}$ Gegen die ausschlaggebende Bedeutung des Aspekts der „Rechtsbewährung“ spricht, dass Notwehr de lege lata nur zur Verteidigung individueller Güter zulässig ist. Warum „die Rechtsordnung“ nur gegen solche Angriffe mit besonderer Vehemenz verteidigt werden darf, nicht aber gegen Angriffe auf kollektive Rechtsgüter wie z. B. eine funktionierende Rechtspflege oder den Schutz der Umwelt, wäre erst zu begründen. ${ }^{9}$

Gegen ein rein individuelles Verständnis der Notwehr wird von den Vertretern des dualistischen Ansatzes insbesondere vorgebracht, dass die besondere „Schneidigkeit“ des Notwehrrechts auf diese Weise nicht erklärt werden könne. ${ }^{10}$ Das Notwehrrecht des $\mathbb{3} 2 \mathrm{StGB}$ reicht bekanntlich sehr weit und umfasst nach ganz h. M. ggf. auch das Recht zur Tötung des Angreifers zur Verteidigung von Sachwerten, ohne, dass es auf die Verhältnismäßigkeit bzw. „Proportionalität“ von angegriffe-

5 Vgl. nur MK-Erb, $\mathbb{3} 32$ Rn. 12; H. Frister GA 1988, 291, 301 ff.; SK-Günther, $\$ 32$ Rn. 12 f.; H. Wagner, Individualistische oder überindividualistische Notwehrbegründung, Berlin 1984; L. Kublen GA 2008, 282 ff.;

A. Engländer, Grund und Grenzen der Nothilfe (Fn. 4), $38 \mathrm{ff}$.

6 E. Schmidhäuser, AT, 2. Aufl., Tübingen1975, 9/86; ders. GA 1991, 97 ff.; s. auch N. Bitzilekis, Die neue Tendenz zur Einschränkung des Notwehrrechts, Berlin 1984.

7 S. nur BGHSt 24, 356, 359; BGH JZ 1973, 252, 253; C. Roxin, Strafrecht Allgemeiner Teil,(Fn. 4), \15 Rn. 3; ders., ZStW 93 (1981), 70.

8 S. nur P. Kasiske Jura 2004, 833.

9 G. Freund, Strafrecht Allgemeiner Teil, 2. Aufl., Berlin2009, $\sqrt{3}$ Rn. 88; A. Engländer, Grund und Grenzen der Nothilfe (Fn. 4),21. Vgl. den Erklärungsversuch bei C. Bertel ZStW 84 (1972), 1, 8, wonach der Bürger zum Schutz der Rechtsgüter des Staates und der Allgemeinheit nur wenig beitragen könne und eine entsprechende Nothilfe letztendlich mehr Schaden als Nutzen bewirken würde. Das wäre noch genauer anhand der einzelnen Delikte zu analysieren, erscheint aber auf den ersten Blick wenig plausibel, weil zumindest Letzteres auch für die (gewaltsame) Verteidigung von Sachwerten gilt. Auch könnte man kaum von einem auch nur in der Mehrzahl der Fälle tatsächlich „ungeeigneten“ Mittel im Vergleich zur völligen Nicht-Intervention ausgehen.

10 Zur Kritik s. C. Roxin, Strafrecht Allgemeiner Teil (Fn. 4), $\$ 15$ Rn. 2; K. Kühl, Strafrecht Allgemeiner Teil, 7. Aufl., München2012, $\$ 7$ Rn. 16 ff. 
nem und verteidigtem Gut ankommen soll. ${ }^{11}$ Weiterhin wird dem Angegriffenen grundsätzlich weder ein Ausweichen, noch das Herbeiholen fremder Hilfe zugemutet. All dies, so heißt es, sei allein mit dem Schutz der bedrohten Güter nicht zu erklären, sondern werde erst vor dem Hintergrund des Aspekts der „Rechtsbewährung “ verständlich. ${ }^{12} \mathrm{Da}$ das Rechtsbewährungsprinzip also in der Diskussion um Grund und Grenzen der Notwehr eine zentrale Rolle spielt, lohnt es sich, diesem bis heute inhaltlich recht unbestimmten Prinzip im Rahmen des vorliegenden Beitrags etwas näher nachzugehen.

\section{B. Die Funktionen des Prinzips der „Rechtsbewährung“}

\section{Notwehrerweiternde Funktion}

Der Aspekt der Rechtsbewährung wird wie gesagt zum einen oft herangezogen, um die besondere Schneidigkeit des Notwehrrechts zu erklären. Auf eine Abwägung der betroffenen Güter soll es aus Gründen der „Rechtsbewährung“ nicht ankommen, ${ }^{13}$ und die für den Angreifer regelmäßig schonenderen Handlungsalternativen des Ausweichens oder der Inanspruchnahme fremder Hilfe werden dem Verteidiger grundsätzlich nicht zugemutet, auch wenn sich der Schutz der unmittelbar attackierten Rechtsgüter auf diese Weise gleich gut oder sogar besser verwirklichen ließe. Dem Aspekt der „Rechtsbewährung “ kommt hier also eine notwehrerweiternde Funktion zu. Vor allem auf diesen besonders charakteristischen (um nicht zu sagen: eigentümlichen) Aspekt des deutschen Notwehrrechts werden die folgenden Ausführungen konzentriert sein.

\section{Notwehrbeschränkende Funktion}

Interessanterweise werden zugleich die weitgehend anerkannten sogenannten „sozialethischen Einschränkungen“ als Fälle fehlender "Gebotenheit" der Notwehr ${ }^{14}$ ganz wesentlich mit dem Hinweis auf das Grundprinzip der „Rechtsbewährung“ erklärt, das in diesem Fall also eine notwehrbeschränkende (damit zugleich: strafbarkeitserweiternde) Funktion erfüllt.

\section{Krasses Missverhältnis}

Bestes Beispiel ist die Einschränkung der Gebotenheit bei besonders „krassem Missverhältnis “ der auf beiden Seiten betroffenen Rechtsgüter. Das schneidige und grund-

11 Ganz h. M., s. nur C. Roxin, Strafrecht Allgemeiner Teil (Fn. 4), $\mathbb{1 5}$ Rn. 2; aus der Rechtsprechung s. nur BGH NStZ 2003, 425; weitere Nachweise bei J. Bülte, GA 2011, 145 f..

12 Vgl. nur H. Schröder JR 1962, 188; T. Lenckner JZ 1973, 254; C. Roxin, Strafrecht Allgemeiner Teil (Fn. 4), $\mathbb{1 5}$ Rn. 47 und 49; OK-Momsen, $\$ 32$ Rn. 33 a.

13 Vgl. die Darstellung bei G. Freund, Strafrecht Allgemeiner Teil (Fn. 9), $\$ 3$ Rn. 85 ff.

14 S. nur L. Kublen GA 2008, 284; C. Roxin, Strafrecht Allgemeiner Teil (Fn. 4), $\$ 15$ Rn. 55 ff. Dabei wird nicht verkannt, dass nicht nur die Einzelheiten sehr umstritten sind, sondern diese Einschränkungen von manchen Autoren insgesamt wegen einer fehlenden eindeutigen gesetzlichen Grundlage für verfassungswidrig gehalten werden, s. die Nachweise bei C. Roxin, Strafrecht Allgemeiner Teil (Fn. 4), $\$ 15$ Rn. 56. 
sätzlich abwägungsfreie Notwehrrecht soll hier - wenn auch nur in Extremfällen doch eine Einschränkung erfahren. Ein Beispiel aus der Rechtsprechung ist etwa der Schuss auf einen Dieb, der mit einer Beute im Wert von (damals) 10 Pfennig auf der Flucht war. ${ }^{15}$ In solchen Fällen, so Roxin als Vertreter eines dualistischen Ansatzes, sei das Interesse an Rechtsbewährung aufgrund der nur geringfügigen Beeinträchtigung des Angegriffenen deutlich herabgesetzt. Das könne man auch daran erkennen, dass der Gesetzgeber hier, wie sich z. B. an den Vorschriften über die Opportunitätseinstellungen in $\mathbb{S} \mathbb{S} 153 \mathrm{ff}$. StPO zeige, eine „Prävention durch Sanktionierung“ nicht als schlechthin unerlässlich ansehe. ${ }^{16}$ Hier wird also offensichtlich eine Parallele von Notwehrhandlung und staatlicher Strafe gezogen. Das liegt auch durchaus nahe, wenn man mit „Rechtsbewährung“ durch Notwehr argumentiert, müsste dann aber, wie später gezeigt wird, zu einer strengeren Begrenzung des Notwehrrechts führen, als dies bislang von den Vertretern eines dualistischen Ansatzes gesehen wird. Diese halten auch hier an der prinzipiellen Härte der zulässigen Verteidigung fest. Da man es in den Bagatellfällen, so wiederum Roxin, nur mit einem „geminderten “ und nicht mit einem völlig beseitigten Rechtsbewährungsinteresse zu tun habe, sei hier das Notwehrrecht grundsätzlich gegeben; eine Schonung des Angreifers sei nur im Hinblick auf dessen Leben aufgrund des „Gebots der Rücksichtnahme“ angezeigt. ${ }^{17}$ Noch weiter geht Schmidhäuser als Vertreter eines rein überindividuellen Ansatzes, nach dem der erwachsene Obstdieb, der trotz Warnung nicht vom Apfelbaum steigt, erschossen werden darf. Dies sei Teil des unerlässlichen „Kampfs ums Recht“, der nun einmal von der „Verteidigungsbereitschaft aller Rechtsgenossen “ abhänge. ${ }^{18}$ Dass viele „Rechtsgenossen“ das glücklicherweise anders sehen, wird später anhand empirischer Daten gezeigt werden. ${ }^{19}$

\section{Provokation}

Auch die Einschränkung des Notwehrrechts nach vorangegangener Provokation wird oft mit dem fehlenden „Rechtsbewährungsinteresse“ begründet, ${ }^{20}$ zumindest

15 OLG Stuttgart, DRZ 1949, 42.

16 C. Roxin, Strafrecht Allgemeiner Teil (Fn. 4), \$15 Rn. 83.

17 C. Roxin, Strafrecht Allgemeiner Teil (Fn. 4), \15 Rn. 84.

18 E. Schmidhäuser, Über die Wertstruktur der Notwehr, in: Göttinger Rechtswissenschaftliche Fakultät (Hrsg.), Festschrift für Honig, Göttingen 1970, 185, 198.

19 S. unten D. II.

20 Ablehnend C. Bertel ZStW 84 (1972), 1, 13: das generalpräventive Interesse an der Verhinderung solcher Angriffe sei genauso gegeben, das zu missbilligende Verhalten des Provokateurs sei für das Notwehrrecht irrelevant. Ebenfalls ablehnend W. Mitsch GA 1986, 533, der entscheidend auf die „Selbstgefährdungskomponente“ des Angriffs abstellt. Der Angreifer könne sich durch Abbruch des Angriffs selbst vor gefährlichen Verteidigungsmaßnahmen schützen. Vor diesem Hintergrund seien die anderen Einschränkungsgründe (Schuldlose, Kinder) zu erklären; letztlich wird eine Anlehnung an die Kriterien der Einwilligung erwogen, W. Mitsch a.a.O., 546 - allerdings müsste doch dann an sich auch die Einwilligungssperre von $\$ 216$ StGB greifen. 
ergänzend zu einer eher individualschutzorientierten Argumentation. ${ }^{21}$ Allerdings sind die Ausführungen hier teilweise etwas vage. So meint der BGH in einer Entscheidung ohne nähere Begründung, das Allgemeininteresse komme „weniger nachdrücklich zur Geltung“, wenn der Verteidiger selbst vorwerfbar zum Angriff beigetragen habe. ${ }^{22}$ Nur wenig konkreter wird Lenckner, der zwei Gesichtspunkte nennt: die Provokation könne erstens den Angriff in einem „milderen Licht“ erscheinen lassen. Zweitens könne die Verteidigungshandlung als Mittel der „Rechtsbewährung “ diskreditiert sein, wenn sie selbst noch im Zeichen des zuvor begangenen Unrechts stehe. Hier kommt der Gedanke einer Art „Bemakelung“ von Verteidiger oder Verteidigungshandlung ins Spiel, der später noch kritisch zu hinterfragen sein wird. ${ }^{23}$

\section{Enge persönliche Verbundenheit von Angreifer und Verteidiger}

Eine weitere, wenn auch sehr umstrittene Fallgruppe ist die Einschränkung des Notwehrrechts, wenn zwischen Angreifer und Verteidiger eine enge persönliche Verbundenheit herrscht, etwa eine familiäre Beziehung. In diesen Fällen, wo besondere Garantenpflichten i. S. v. $\mathbb{S} 13$ StGB bestehen, trete das Rechtsbewährungsinteresse hinter der Pflicht zu menschlicher Rücksichtnahme zurück. ${ }^{24}$ Möglicherweise steht dahinter zugleich die Überlegung, dass solche Konstellationen eher privaten Charakter haben, nur die unmittelbar Beteiligten „etwas angehen“ und keinen überindividuellen Handlungsbedarf erzeugen. ${ }^{25}$ Beides leuchtet allerdings nicht recht ein eine Handlungspflicht zur Rettung des anderen kann doch kaum herangezogen werden, um zu begründen, dass man ausgerechnet von dieser Person einen Angriff in größerem Umfang dulden muss. Man könnte ganz im Gegenteil gerade innerhalb solcher Beziehungen den Angriff als besonders schwerwiegendes (und aufgrund offensichtlicher Wiederholungsgefahr auch gefährliches) Geschehen werten, was für eine uneingeschränkte Verteidigungsmöglichkeit spräche. Auch dass hier nur ein begrenztes überindividuelles Interesse an der generellen Verhinderung bzw. Verfolgung solcher Taten besteht, ist nicht überzeugend - dem widerspricht es z. B., dass Nr. 233 der Richtlinien für das Straf- und Bußgeldverfahren gerade bei Körperverletzungen zwischen Personen mit enger persönlicher Verbundenheit eine Bejahung

21 S. etwa C. Roxin ZStW 93 (1981), 86; H. Wagner, Individualistische oder überindividualistische Notwehrbegründung (Fn. 5), 71.

22 BGH JZ 1973, 253. S. auch C. Roxin, Strafrecht Allgemeiner Teil (Fn. 4), $\$ 15$ Rn. 64. A. A. C. Schöneborn NStZ 1981, 101 ff., der auf die Gemütslage des Provozierten abstellt.

23 S. unten C. III. 2.

24 C. Roxin, Strafrecht Allgemeiner Teil (Fn. 4), \$15 Rn. 93.

$25 \mathrm{Zu}$ Recht kritisch gegen eine solche denkbare Argumentation G. Freund, Strafrecht Allgemeiner Teil (Fn. 9), \$ 3 Rn. 123: Auch hier sei eine überindividuelle Bedeutung gegeben (was ja schon die Strafbarkeit zeige). Zieht man die Parallele zur generalpräventiven Funktion der Strafe (s. unten), ist das nur konsequent. Allenfalls könnte man im Bereich der reinen Privatklagedelikte eine Einschränkung des Notwehrrechts bzw. eine Reduzierung auf reinen Individualschutz ohne Rechtsbewährungskomponente begründen. 
des „öffentlichen Interesses“ an der Strafverfolgung vorsieht. Es ist daher nicht verwunderlich, dass besonders diese Notwehreinschränkung sehr umstritten ist.

\section{Angriffe von schuldlos Handelnden}

Eine im Grundsatz dagegen einhellig anerkannte Einschränkung des Notwehrrechts ist die Fallgruppe der Angriffe, die von schuldlos handelnden (etwa volltrunkenen) Erwachsenen oder von strafunmündigen Kindern ausgehen. Auch hier, wo die Intensität des Angriffs auf die individuellen Güter durchaus genauso schwerwiegend sein kann wie bei einem voll schuldhaft Handelnden, basiert die Begründung der Notwehreinschränkung zentral auf einem fehlenden oder zumindest eingeschränkten Interesse an Rechtsbewährung. ${ }^{26}$ Der Angriff werde hier, so meint Schröder, nicht in vollem Umfang von der Rechtsordnung missbilligt; ${ }^{27}$ das Recht habe hier „keinen Anlass, sich zu bewähren“. ${ }^{28}$ Das soll nach Roxin auch bei lediglich verminderter Schuldfähigkeit gem. $\mathbb{} 21$ StGB gelten: Die wesentliche Herabsetzung strafrechtlicher Sanktionen in diesen Fällen sei ein Zeichen für ein gemindertes „Rechtsbewährungsinteresse“, daher sei hier auch von Seiten des Verteidigers Rücksicht angezeigt. ${ }^{29}$ Auch hier wird also eine Parallele von Notwehr und strafrechtlicher Sanktionierung (als originär staatlicher Aufgabe) gezogen.

\section{Notwehr gegen Schweigegelderpressung}

Eine mögliche Einschränkung des Notwehrrechts wird schließlich noch in den Fällen der Notwehr gegen eine Schweigegelderpressung diskutiert. Bei dieser sog. „Chantage" droht der Erpresser damit, kompromittierende (wahre) Tatsachen, etwa die Begehung einer Straftat, publik zu machen, wenn der Betroffene nicht auf seine in der Regel finanziellen Forderungen eingeht. Diese erpresserische Drohung bewirkt nach ganz h. M. einen andauernden Angriff auf Willensfreiheit und Vermögen des Opfers, so dass eine Notwehrlage vorliegt. Die Einschaltung der Polizei als milderes Mittel im Vergleich zur auch gewaltsamen Gegenwehr sei dem Erpressten nicht zumutbar, da dann die von ihm verheimlichten Tatsachen ans Licht kämen. Dennoch dürfe sich der Erpresste nicht mit voller Härte wehren: Zumindest gewaltsame Gegenwehr, insbesondere die Tötung des Erpressers sei ausgeschlossen. Und auch dieses

26 C. Roxin, Strafrecht Allgemeiner Teil (Fn. 4)\$15 Rn. 61; H.H. Jescheck/T. Weigend, Strafrecht Allgemeiner Teil, 5. Auf., Berlin 1996, $\$ 32$ III 3 a).

27 H. Schröder JR 1962, 188.

28 H. Schröder JR 1962, 189. A. A. G. Freund, Strafrecht Allgemeiner Teil (Fn. 9), $\mathbb{S} 3$ Rn. 111, der auf die Tatsache hinweist, dass auch bei Volltrunkenen oder fahrlässig Angreifenden eine Strafe erfolge, daher doch ein Angriff auf die Rechtsordnung angenommen werde. Das ist aber nicht zwingend: Einerseits kann man mit einem zumindest (anerkannt) reduzierten Bedürfnis nach „Rechtsbewährung“ in solchen Fällen argumentieren. Andererseits knüpfen die Bestrafungsmöglichkeiten der actio libera in causa bzw. des $\$ 323$ a StGB, auf die Freund hier offenbar anspielt, gerade nicht an das mit der unmittelbaren Verletzungshandlung ausgelöste Strafbedürfnis an, sondern an ein entsprechendes Vorverhalten (jedenfalls, sofern man bei der actio libera in causa mit der h. M. dem Tatbestands- bzw. Vorverlagerungsmodell folgt, krit. zur Konstruktion demnächst SSW-Kaspar, 2. Aufl., \$20 Rn. 102 f.).

29 C. Roxin, Strafrecht Allgemeiner Teil (Fn. 4), \15 Rn. 64. 
Ergebnis wird maßgeblich auf Überlegungen zur „Rechtsbewährung“ gestützt; 30 zum einen wird darauf verwiesen, dass der Erpresste hier in vielen Fällen selbst vorwerfbar gehandelt habe - wobei nicht immer ganz klar wird, ob dass seine individuelle Schutzwürdigkeit herabsetzen oder seine persönliche Eignung als Verteidiger der Rechtsordnung in Frage stellen soll. Auf dieses „personale Element“ der Rechtsbewährung wird später noch eingegangen. ${ }^{31}$ Interessant für die weiteren Überlegungen im Hinblick auf den Inhalt des Rechtsbewährungsprinzips ist auch der Gesichtspunkt der v. a. von Amelung vorgebracht wird: Der Erpresste müsse hier, um die Aufdeckung der Hintergründe der Erpressung zu verhindern, notgedrungen „heimlich“ vorgehen. Dann sei aber zu befürchten, dass bei der Allgemeinheit (etwa nach dem Auffinden der Leiche des Erpressers) der - falsche - Eindruck entstünde, man habe es mit einer ganz gewöhnlichen Gewalttat zu tun. In einem solchen Fall diene die Verteidigung dann nicht der „Rechtsbewährung“, sondern führe geradezu zu einer „Erschütterung des Rechtsfriedens“ und sei daher abzulehnen. ${ }^{32}$

\section{Kritik am Prinzip der „Rechtsbewährung“}

Damit ist man bei möglichen Kritikpunkten angelangt. Das betrifft zunächst die Frage des historischen Hintergrundes dieses Prinzips, darüber hinaus den Vorwurf der Zirkularität und (damit zusammenhängend) der inhaltlichen Unbestimmtheit.

\section{Historischer Hintergrund}

Schon die Herleitung des Rechtsbewährungsprinzips in historischer Sicht ist alles andere als klar. Oft findet sich lediglich der Verweis auf die vielzitierte Formulierung von Berner aus dem Jahre 1848, wonach eine Beschränkung des Notwehrrechts nicht in Frage komme: „Es wäre Unrecht, wenn das Recht dem Unrecht weichen müsste “. ${ }^{33}$ Dieses mittlerweile geflügelte Wort wird schon früh in mehreren Entscheidungen des Reichsgerichts aufgegriffen ${ }^{34}$ und wird bis heute (möglicherweise zu Unrecht $)^{35}$ zur Begründung des Rechtsbewährungsprinzips herangezogen. ${ }^{36}$ Liest man allerdings die gesamte Passage von Berner, in der der berühmte Satz fällt, so zeigt sich schnell, dass er dort (auf der Grundlage Hegelianischen Denkens) von Voraussetzungen ausgeht, die heutigen normativen Maßstäben eindeutig widersprechen. ${ }^{37}$

30 S. nur C. Roxin, Strafrecht Allgemeiner Teil (Fn. 4), $\$ 15$ Rn. 100; K. Amelung GA 1982, 398. Zum Ganzen auch J. Kaspar GA 2007, 36.

31 S. unten C. III. 2.

32 K. Amelung GA 1982, 398, 392 ff.; s. auch C. Roxin, Strafrecht Allgemeiner Teil (Fn. 4), Rn. 100.

33 A. F. Berner Archiv des Criminalrechts 1848, 547.

34 S. RGSt 21, 168, 174; 55, 82, 85.

35 Vgl. A. Engländer, Grund und Grenzen der Nothilfe (Fn. 4), 22 m. w. N., der davon ausgeht, dass Berner selbst eine individualistische Position vertrat.

36 Nachweise bei J. Bülte GA 2011, 148.

37 S. auch die kritische Auseinandersetzung bei H. Koriath, Einige Gedanken zur Notwehr, in: G. Britz u. a. (Hrsg.), Festschrift für Müller-Dietz, München 2001, 361, 363 ff. 
Bei der Notwehr gehe es - anders als etwa beim Notstand - nicht um Rechtspositionen auf beiden Seiten, die zu berücksichtigen und ggf. abzuwägen seien. Vielmehr stünden sich hier schlicht „Recht“ (in Person des Verteidigers) und „Unrecht“ (in Person des Angreifers) gegenüber. „Es ist in der Notwehr jedes Recht“, so Berner ausdrücklich, „welches sich gegen jedes Unrecht unbedingt verteidigen darf“..38 Der Angreifer, so muss man Berner hier wohl verstehen, kann überhaupt keine rechtlichen Interessen geltend machen. Er ist vogelfrei, ein „outlaw“, in moderner Terminologie: nicht mehr Bürger, sondern „Feind“. ${ }^{39}$ Das widerspricht aber ganz offensichtlich den Maßstäben des Verfassungsrechts, das nur unter engen Voraussetzungen eine „Verwirkung“ von Grundrechten vorsieht, staatliche Schutzpflichten gerade im Hinblick auf das menschliche Leben enthält und die Menschenwürde als unverzichtbare hochrangige Rechtsposition anerkennt, deren Schutz man durch noch so verwerfliches Verhalten nicht verliert. Die von Berner bei seiner Äußerung zugrunde gelegte Prämisse der vollständigen „Rechtlosigkeit“ des Angreifers aufgrund seiner Stellung quasi als „personifiziertes Unrecht“ kann also nicht überzeugen.

Ohnehin kommt hier ein holzschnittartiges Schwarz-Weiß-Denken zum Tragen, das den Vorstellungen der damaligen Zeit entsprochen haben mag, aus heutiger aufgeklärter Sicht aber (auch im Hinblick auf kriminologische Erkenntnisse) nicht mehr haltbar ist. Die in gewisser Weise beruhigende und sozialpsychologisch vermutlich bis heute überaus wirkmächtige gedankliche Aufteilung der Gesellschaft in „Verbrecher" einerseits und „anständige Bürger“ andererseits ist an sich durch die Ergebnisse von Dunkelfeldstudien nachhaltig erschüttert. Kriminalität ist eben kein pathologisches Phänomen bei einigen wenigen, sondern tritt jedenfalls in Form von leichter bis mittelschwerer Delinquenz bei nahezu allen (vor allem jüngeren) Menschen auf. ${ }^{40}$ Umso mehr ist Skepsis angebracht gegenüber einer radikalen Versagung von rechtlichem Schutz gegenüber einer Person allein aufgrund ihres rechtswidrigen Verhaltens.

\section{Zirkularität}

Darüber hinaus ist gegen ein auf diese Weise begründetes Prinzip der „Rechtsbewährung “ der Einwand zu erheben, dass dabei die Gefahr einer zirkulären Argumentation besteht, und zwar in mehrfacher Weise.

Zunächst wäre es offensichtlich nicht zulässig, pauschal zu behaupten, der Angegriffene repräsentiere gerade auch mit der von ihm gewählten konkreten Verteidigungshandlung „das Recht“. Denn ob er sich mit seiner Verteidigung im Rahmen der von $\mathbb{} 32$ StGB gezogenen Grenzen und damit innerhalb „des Rechts“ bewegt,

38 A. F. Berner Archiv des Criminalrechts 1848, 547, 554.

$39 \mathrm{Zu}$ der von Jakobs angestoßenen Diskussion um ein vom „Bürgerstrafrecht“ zu unterscheidendes „Feindstrafrecht" s. nur umfassend L. Greco, Feindstrafrecht, Baden-Baden 2010 m. w. N.

40 S. B. D. Meier, Kriminologie, 4. Aufl., München2010, $\$ 5$ Rn. 60 f. 
ist ja gerade die Frage, die es noch zu beantworten gilt. ${ }^{41}$ In einer Notwehrkonstellation kann es ohne weiteres dazu kommen, dass auf das „Unrecht“ des Angriffs mit dem „Unrecht“ einer nicht von $\$ 32$ StGB gedeckten Verteidigungshandlung reagiert wird. Das liegt auch der Regelung zum Notwehrexzess in $\$ 33$ StGB zugrunde, die in bestimmten Konstellationen (lediglich) eine Entschuldigung des Verteidigers vorsieht, was voraussetzt, dass die Notwehrhandlung rechtswidrig war. Allein mit dem Schlagwort vom Kampf „des Rechts“ gegen „das Unrecht“ kommt man also nicht weiter. ${ }^{42}$ Gleiches gilt für die oft als Kehrseite der „Rechtsbewährung“ und als Begründung für „fehlende Gebotenheit“ bemühte Wendung vom „Rechtsmissbrauch“. Denn ob der Verteidiger das Notwehrrecht „missbraucht“, hängt entscheidend von der eigentlich zu beantwortenden Frage der Reichweite eben dieses Notwehrrechts ab. Ohnehin handelt es sich dabei um einen letztlich verzichtbaren Begriff; denn wer sich außerhalb des Bereichs zulässiger Notwehr bewegt, „missbraucht“ kein an sich existierendes Notwehrrecht, sondern hat schlicht keines. ${ }^{43}$

Zirkulär ist auch die oft anzutreffende Vorgehensweise, dass zur Begründung des Rechtsbewährungsprinzips auf die überwiegend anerkannten Inhalte des Notwehrrechts verwiesen wird. Sowohl die sogenannten „sozial-ethischen Einschränkungen“ einerseits als auch die „Schneidigkeit“ des Notwehrrechts erscheinen dann als Beleg für die Geltung des Rechtsbewährungsprinzips, gleichzeitig werden aber gerade diese Inhalte des Notwehrrechts (wie oben gezeigt) ihrerseits auf die Grundprinzipien der Notwehr und damit auch das Rechtsbewährungsprinzip zurückgeführt. ${ }^{44}$

Versteht man die Redewendung vom „Kampf des Rechts gegen das Unrecht“ dagegen nur als Hinweis auf die Tatsache, dass der Angreifer rechtswidrig handelt, während der Angegriffene zur Verteidigung einer ihm (oder einem Dritten) legitimerweise zustehenden Rechtsposition handelt (und insofern im Ausgangspunkt „im Recht“ ist), so wäre das lediglich eine Umschreibung der gesetzlichen Voraussetzungen der Notwehrlage, die als solche noch kein Argument für die Reichweite der Notwehrbefugnis enthalten.

41 Zutreffend H. Koriath, FS Müller-Dietz (Fn. 38), 361, 366 sowie 371 mit Hinweis auf K. Seelmann ZStW 89 (1977), 36, 45.

42 Vgl. K. Bernsmann ZStW 104 (1992), 290, 316 ; A. Engländer, Grund und Grenzen der Nothilfe (Fn. 4), 24; s. auch P. Kasiske Jura 2004, 833, nach dem es sich um einen „inhaltlichen Teilaspekt“ der Notwehr handelt, der aber keine Begründung enthalte.

43 Vgl. H. Koriath, FS Müller-Dietz (Fn. 38), 361, 382.

44 S. den Hinweis von P. Kasiske Jura 2004, 833 auf A. Hoyer JuS 1988, 91. 


\section{Inhaltliche Unbestimmtheit}

Die Argumentation mit „Rechtsbewährung“ droht so lange zirkulär und inhaltsleer zu bleiben, solange man keine genaueren Maßstäbe benennen kann, was genau man mit diesem Begriff meint. ${ }^{45}$

\section{1. „Rechtsbewährung“ als Einfalltor subjektiver Wertungen}

Daran fehlt es in vielen Fällen. Man kann sich stattdessen des Eindrucks nicht erwehren, dass das jeweils für sachgerecht angesehene Ergebnis aus dem Rechtsbewährungsprinzip abgeleitet wird, nachdem man es zuvor mit dem entsprechenden Inhalt angefüllt hat. „Rechtsbewährung“ erscheint dann als bloße Worthülse, als „Passepartout“ einer von eigenen subjektiven Wertungen geleiten Argumentation. Ein Indiz dafür ist, dass wie erwähnt sowohl das prinzipielle Fehlen von Verhältnismäßigkeitserwägungen als auch die Begrenzung des Notwehrrechts bei krassem Missverhältnis der betroffenen Rechtsgüter gleichermaßen mit „Rechtsbewährung“ begründet wird. Die Bewährung des Rechts gestattet nach dieser Ansicht, dass besonders scharf und „unverhältnismäßig“ gegen rechtswidrige Angriffe vorgegangen wird - fordert aber dann doch ein gewisses Maßhalten. Das geht aber oft nicht über den Charakter einer bloßen Behauptung hinaus - Koriath spricht in diesem Zusammenhang nicht zu Unrecht von der Degeneration des Rechtsbewährungsprinzips zu einem „argumentativen Trick“. 46

\section{Personales Element?}

Die Unklarheiten über die mit Notwehr anzustrebende „Rechtsbewährung“ zeigen sich auch im Hinblick auf das oben bereits erwähnte „personale Element“, also die (regelmäßig nicht näher begründete) Annahme, dass bestimmte negativ bewertete persönliche Umstände in der Person des Verteidigers seine Handlung mit einem Makel versehen und ihr damit den Charakter als „rechtsbewährend“ nehmen können. ${ }^{47}$ Gerade das oben genannte Beispiel des Chantage-Falles macht das sehr deutlich. Der Erpresste hat selbst etwas zu verheimlichen - und soll u. a. deswegen in seinem Notwehrrecht beschränkt sein. Auch die Fallgruppe der vorwerfbaren Provozierung der Notwehrlage ließe sich hier einordnen. Konsequent zu Ende gedacht würde das aber bedeuten, dass übel beleumundete Personen, vor allem Vorbestrafte, generell vom Notwehrrecht auszunehmen wären. ${ }^{48}$ Der unlängst erfolgte Freispruch eines Mitgliedes der berühmt-berüchtigten „Hells Angels“ wegen Notwehr (bzw. im konkreten Fall: Putativnotwehr) ${ }^{49}$ wäre dann von vornherein ausgeschlossen. Das

45 Zum Problem der Unbestimmtheit s. G. Freund, Strafrecht Allgemeiner Teil (Fn. 9), $\mathbb{3}$ Rn. 86; H. Koriath, FS Müller-Dietz (Fn. 38), 361, 368 sowie 371 ff. S. auch den Hinweis von L. Kublen GA 2008, 287 auf die Flexibilität des Prinzips.

46 H. Koriath, FS Müller-Dietz (Fn. 38), 369.

47 Vgl. ausdrücklich C. Roxin ZStW 75 (1963), 567. So auch P. Kasiske Jura 2004, 838.

48 So G. Freund, Strafrecht Allgemeiner Teil (Fn. 9), $\$ 3$ Rn. 119.

49 BGH NStZ 2012, 272. 
erscheint wenig sachgerecht - warum sollte der rechtswidrig Angreifende von eventuellen Verfehlungen des Angriffsopfers profitieren ${ }^{50}$ ? Insofern ist es zu begrüßen, dass der BGH in seiner Entscheidung zur Notwehr gegen einen Erpresser in dieser Frage klar Stellung bezogen hat. Er führt aus:

„Auch demjenigen, der früher eine strafbare Handlung begangen hat, steht grundsätzlich ein uneingeschränktes Notwehrrecht zur Seite, wenn er in anderem Zusammenhang selbst Opfer einer Straftat wird. Er hat nicht etwa deshalb, weil die gegen ihn gerichtete Tat (hier: eine Erpressung) vom Täter an seine eigene gegen die Rechtsgüter Dritter begangene eigene Straftat angeknüpft wird, einen 'Status minderen Rechts'".51

Das gilt natürlich, wie man hinzufügen kann, erst recht für völlig andere Taten, die in gar keinem Zusammenhang mit dem Angriff stehen. Da der Senat hier vom Notwehrrecht insgesamt spricht und dabei ausdrücklich eine dualistische Ansicht zugrunde legt ${ }^{52}$ erteilt er damit auch zugleich einer Beschränkung des Rechtsbewährungsprinzips wegen eines „persönlichen Makels“ des Verteidigers eine Absage. ${ }^{53}$

\section{Rechtsordnung als „Schutzgut" der Notwehr}

In der oft synonym zur „Rechtsbewährung“ verwendeten Bezeichnung der „Verteidigung der Rechtsordnung“ kommt ein weiteres Problem des Rechtsbewährungsprinzips deutlich zum Ausdruck. Es geht um die Frage, inwiefern überhaupt „die Rechtsordnung “ als solche Schutzgut der Notwehr sein kann.

Denn der genaue Bezugspunkt ist nicht eindeutig: Soll es um die Bewährung sämtlicher Normen der Rechtsordnung gehen? Das würde dann auch $\$ 32$ StGB umfassen, was erneut in einen Zirkel führt; denn ob die Verteidigungshandlung geeignet ist, auch die Geltung der Notwehrregelung symbolisch zu bekräftigen, hängt ja ersichtlich davon ab, dass dabei die Grenzen legitimer Notwehr eingehalten werden. Dann wäre es erneut unmöglich, aus dem Gedanken der Rechtsbewährung seinerseits Anhaltspunkte für eben diese Grenzen abzuleiten. Benötigt würden dafür externe Maßstäbe. Man könnte nun auf die Bewährung sonstiger Normen der Rechtsordnung insgesamt abstellen. Aber wird z. B. durch einen Diebstahl wirklich „die Rechtsordnung “ im Ganzen in Frage gestellt ${ }^{54}$ ? Oder nur das Verbot des $\mathbb{2} 242$ StGB? Geht

50 Vgl. C. Bertel ZStW 84 (1972), 1, 13: „Durch die Notwehr schützt sich die Rechtsordnung um der Rechtssicherheit willen, ganz gleich, ob der Angegriffene ein Heiliger oder ein Verbrecher ist". S. auch W. Mitsch GA 1986, 541 mit dem Hinweis auf $\$ 24$ II, der zeige, dass auch die Verhinderungsleistung des vorsätzlich Handelnden honoriert werde; insofern sei der Verlust der Legitimation des Angegriffenen zur „Verteidigung der Rechtsordnung“ nicht plausibel.

51 BGH NStZ 2003, 425.

52 BGH NStZ 2003, 425, 427.

53 Ablehnend auch L. Kublen GA 2008, 293; C. Roxin ZStW 93 (1981), 76.

54 Vgl. auch H. Koriath, FS Müller-Dietz (Fn. 38), 361, 372, der diese Vorstellung als ,schlicht hysterisch" bezeichnet; ablehnend auch A. Engländer, Grund und Grenzen der Nothilfe (Fn. 4), 27. 
man von aber Letzterem aus, wäre erst zu begründen, warum das beeinträchtigte „Rechtsbewährungsinteresse“ vom Gewicht des jeweils bedrohten Rechtsgutes jedenfalls grundsätzlich abgekoppelt sein sollte. Denn wiegt der Angriff auf das Tötungsverbot nicht stets schwerer als jener auf das Verbot, andere zu bestehlen? Und müsste man dann nicht stets von einem abgestuften Gewicht des Rechtsbewährungsinteresses je nach Art und Intensität des Angriffs ausgehen ${ }^{55}$ ?

Will man dagegen tatsächlich auf den Schutz „der Rechtsordnung“ insgesamt abstellen und diesem eine so zentrale Rolle für die Reichweite der Notwehr einräumen, mag das nicht recht dazu passen, dass Notwehr im Übrigen zum Schutz kollektiver Rechtsgüter gerade nicht geübt werden darf. Auch ist es eine offene Frage, warum ausgerechnet der Verhältnismäßigkeitsgrundsatz als zentraler Bestandteil der Rechtsordnung von dieser Bewährungsfunktion ausgenommen sein sollte. ${ }^{56}$ Ein denkbares Argument hierfür wäre, dass eine unmittelbare Drittwirkung der Grundrechte und damit auch des Verhältnismäßigkeitsgrundsatzes zwischen den Bürgern nach ganz herrschender Ansicht nicht besteht. Die Abwehrfunktion der Grundrechte, und damit auch der überwiegend aus dem Wesen der Grundrechte abgeleitete Grundsatz der Verhältnismäßigkeit, wären dann aufgrund des rein privaten Charakters der Notwehrhandlung gar nicht einschlägig. Wie im Folgenden gezeigt wird, ist eine solche Argumentation aber jedenfalls dann nicht konsequent, wenn man auf dem überindividuellen Aspekt der Notwehr besteht; denn dann handelt es sich bei der Verteidigungshandlung nicht mehr nur um eine rein private Angelegenheit zwischen zwei Bürgern.

\section{Rechtsbewährung als generalpräventive Komponente der Notwehr?}

Diese Überlegung vom auch öffentlichen Charakter der Verteidigungshandlung muss noch etwas vertieft werden; das führt nach hier vertretener Ansicht zur einzigen Möglichkeit, dem Rechtsbewährungsprinzip eigenständigen inhaltlichen Gehalt zu verleihen, der dem beschriebenen Vorwurf der Zirkularität und Unbestimmtheit, wenn nicht sogar Beliebigkeit entgeht. Es handelt sich um die Vorstellung, dass die Aussicht auf (auch massive) Verteidigungshandlungen potenzielle Angreifer von vorn herein von ihren Taten abhalten soll. Hinzu kommt der Gedanke einer positiven Wirkung in der Gesellschaft, wenn sich „die Rechtsordnung“ schon im Rahmen der Verteidigungshandlung bewährt. Man bewegt sich dann ganz offensichtlich im Kontext der Generalprävention in ihrer sowohl negativen, abschreckenden wie auch ihrer positiven, auf Wiederherstellung des Rechtsfriedens gerichteten Komponente.

55 So auch A. Engländer, Grund und Grenzen der Nothilfe, $27 \mathrm{f}$.

56 Zutreffend J. Bülte GA 2011, 158; ähnlich bereits H. Koriath, FS Müller-Dietz (Fn. 38), 361, 372 sowie K. Bernsmann ZStW 104 (1992), 290, 310. 


\section{Hinweise für ein solches Verständnis von Rechtsbewährung}

Auch wenn der Zusammenhang nicht immer explizit gemacht oder gar ausführlich begründet wird, gibt es doch zahlreiche Hinweise auf die Parallelen von Rechtsbewährung und Generalprävention. So ist schon ganz auffällig, dass der generalpräventive Aspekt der Strafe im Gesetz in mehreren Vorschrift als „Verteidigung der Rechtsordnung“ erscheint, und exakt diese Begriffe werden auch oft als Umschreibung des Effekts der „Rechtsbewährung “ durch Notwehr verwendet. ${ }^{57}$ Das lässt sich bereits anhand des Regierungsentwurfs von 1962 zeigen. Das Notwehrrecht, so heißt es dort, schrecke „in wirksamer Weise allgemein davon ab, Unrecht zu tun“; 58 Angriffe auf individuelle Güter seien „zugleich Angriffe gegen die Rechtsordnung als solche, die der Notwehrübende zugleichmitverteidigt".59 Auch anhand der Äußerungen mancher Autoren lässt sich dies belegen: Notwehr sei, so heißt es in einer Abhandlung, vielleicht sogar das wirksamste Mittel, die Häufigkeit von Straftaten in erträglichen Grenzen zu halten; wörtlich heißt es dann: „Für die Generalprävention - den Ausdruck im weitesten Sinn verstanden - ist das Institut der Notwehr unentbehrlich und durch repressive Maßnahmen nicht zu ersetzen". ${ }^{60}$ Auch bei Roxin finden sich weitere, sowohl ausdrückliche als auch implizite Hinweise auf generalpräventive Wirkungen der Notwehr, ${ }^{61}$ nicht zuletzt die oben schon mehrfach erwähnte funktionale Äquivalenz von Verteidigungshandlung und strafrechtlicher Sanktionierung, die an sich nur vor dem Hintergrund generalpräventiven Denkens $^{62}$ plausibel ist - denn dass die Verteidigungshandlung als Mittel der individuellen Besserung bzw. Resozialisierung ausscheidet, liegt auf der Hand, während eine „schuldausgleichende“ oder „vergeltende“ Funktion gegenüber einer Handlung, die u. U. noch gar nicht begonnen wurde, sondern nur „unmittelbar bevorsteht“, ebenfalls nicht in Betracht kommt. Ein solches generalpräventives Verständnis von Rechtsbewährung erscheint gut vertretbar, wirft aber weitere Fragen auf.

\section{Empirisches oder normatives Verständnis von Generalprävention?}

Zunächst müsste klargestellt werden, ob man ein „empirisches“ oder eher „normatives“ Verständnis der generalpräventiven Wirkung der Verteidigungshandlung zugrunde legt. Das dürfte sich mit der Frage decken, ob es - wenn man das Rechtsbewährungsprinzip grundsätzlich anerkennt - dabei um die Verteidigung der empiri-

57 Vgl. A. Engländer, Grund und Grenzen der Nothilfe (Fn. 4), 15 Fn. 32.

58 BT-Drucks. IV/7650, 157.

59 BT-Drucks. IV/7650, 157.

60 C. Bertel ZStW 84 (1972), 1, 10.

61 Vgl. etwa C. Roxin, Strafrecht Allgemeiner Teil (Fn. 4), \$15 Rn. 2; s. dazu auch P. Kasiske Jura 2004, 833 sowie die weiteren Nachweise bei A. Engländer, Grund und Grenzen der Nothilfe (Fn. 4), 15.

62 Wobei man hier den an sich der Spezialprävention zugeordneten Aspekt der individuellen Abschreckung des Angreifers selbst mit einschließen müsste. Denn ist durchaus denkbar, dass dieser von der Fortführung seines Angriffs durch die geleistete Notwehr abgehalten wird. 
schen oder normativen Geltung der Rechtsordnung gehen solle. ${ }^{63}$ Hier scheint allein ein im Ansatz empirisches Verständnis weiterführend, das auf tatsächliche Wirkungen des Notwehrrechts und seiner Ausübung aufbaut. Andernfalls könnte man auf den generalpräventiven Aspekt auch gänzlich verzichten und unmittelbar (beispielsweise) auf die „Angemessenheit“ der Notwehr nach rein normativen Kriterien abstellen. Demgegenüber eröffnet ein empirischer Ansatz die Möglichkeit, inhaltliche Maßstäbe für eine Konturierung des Notwehrrechts zu ermitteln. Allerdings muss man dabei realistisch bleiben. Dass die Notwehrhandlung im Einzelfall in einem bestimmten quantifizierbaren Umfang messbar zur Generalprävention beiträgt, wird man nicht ernsthaft verlangen können, zumal eine solche Feststellung schon im Hinblick auf die Abschreckungsfunktion mit großen methodischen Schwierigkeiten behaftet ist, bei der von Haus aus stärker normativ ausgerichteten positiven Generalprävention aber endgültig an ihre Grenzen stoßen würde. Es kann letztlich also nur um die Frage gehen, ob die gesetzlich vorgesehene Verteidigungshandlung abstraktgenerell tauglich erscheint, Täter von rechtswidrigen Angriffen abzuhalten bzw. den durch den Angreifer erschütterten „Rechtsfrieden“ wiederherzustellen. ${ }^{64}$

Dabei ist nach hier vertretener Ansicht zwingend der tatsächliche Sachverhalt zugrunde zu legen. Denn es geht hier wie bei der Subsumtion eines Geschehens unter einen Straftatbestand um die Fixierung der Grenzen von Recht und Unrecht - und diese muss aus rechtsstaatlichen Gründen (vor allem im Hinblick auf Rechtssicherheit und Gleichheit der Rechtsanwendung) stets auf der Grundlage eines (richterlich festgestellten) tatsächlichen Geschehens beruhen und darf nicht von dem falschen Eindruck abhängen, den die Öffentlichkeit von einem bestimmten Geschehen hat. Eine Einschränkung des Notwehrrechts des Erpressten allein deshalb, weil er seine Verteidigung heimlich begehen wird, ist daher nicht angebracht, so wie ja auch im Übrigen dem Notwehr Übenden nicht auferlegt wird, sich zu melden und die Umstände seiner Verteidigungshandlung aufzuklären.

Darüber hinaus ist zweifelhaft, ob die „Schneidigkeit“ des Notwehrrechts aus einem generalpräventiv verstandenen Rechtsbewährungsprinzip abgeleitet werden kann.

Was den Aspekt der negativen Generalprävention anbelangt, bietet es sich an, auf die Ergebnisse der empirischen Forschung zur generalpräventiven Wirkung der Strafe zurückzugreifen. ${ }^{65}$ Diese lassen sich vereinfacht dahingehend zusammenfassen, dass ein Abschreckungseffekt wenn überhaupt nur von der Existenz einer potenziellen Sanktionierung in gewisser Höhe ausgeht, der Härte der Sanktionierung aber keine

63 Vgl. die instruktive Darstellung bei A. Engländer, Grund und Grenzen der Nothilfe (Fn. 4), 10 ff.

64 Vgl. A. Engländer, Grund und Grenzen der Nothilfe (Fn. 4), 18.

65 Vgl. nur H. Schöch, Empirische Grundlagen der Generalprävention, in: T. Vogler (Hrsg.), Festschrift für Jescheck, Berlin 1985, 1085 ff.; D. Dölling ZStW 102 (1990), 1 ff.; F. Streng, Strafrechtliche Sanktionen, 3. Aufl., Stuttgart 2012, Rn. 59. 
Bedeutung zukommt. Neben anderen Faktoren spielt die vom potenziellen Täter erwartete „Entdeckungswahrscheinlichkeit“ eine Rolle; übertragen auf die Notwehr würde das bedeuten, dass allein die erwartete „Verteidigungswahrscheinlichkeit“66 einen Einfluss haben könnte, nicht aber die erwartete Intensität der Verteidigungshandlung ${ }^{67}$. Besonders drastische Notwehrmaßnahmen mit einem generalpräventiven Effekt zu begründen, ist vor diesem Hintergrund also fragwürdig. Denkbar wäre eine solche Argumentation allenfalls in den Fällen, in denen es um eine völlige Versagung des Notwehrrechts geht, wo dem Angegriffenen also ein kampfloses Ausweichen zugemutet wird oder die Preisgabe des Eigentums, wenn der Dieb ansonsten nur mit tödlicher Gewalt zu stoppen wäre. Aber auch dann bleibt ja als mögliches generalpräventives Auffangnetz die Tatsache der materiellen Strafbarkeit bestehen, mit der Möglichkeit, durch Anzeige des Geschehens eine strafrechtliche Sanktion herbeizuführen. Alles in allem erscheint es rechtstatsächlich wenig plausibel, dass gerade die Schneidigkeit des Notwehrrechts ernsthaft zur Verhinderung von Straftaten beiträgt. In den Bereichen, in denen vor Tatbegehung überhaupt eine Art Reflexion bzw. „Kosten-Nutzen-Rechnung“ erfolgt, wird der Täter, der im konkreten Fall mit ernsthafter Gegenwehr rechnet, ebenso tendenziell von der Tat Abstand nehmen wie der Täter, der im konkreten Fall mit der sehr realistischen Möglichkeit einer Sanktionierung rechnet - jeweils unabhängig von deren Intensität.

Hinzu kommt, dass die Schärfe des Notwehrrechts nicht sehr bekannt zu sein scheint. ${ }^{68}$ Die empirische Dresdener Notwehrstudie von Amelung und Kilian hat vielmehr gezeigt, dass die Bevölkerung überwiegend und ganz selbstverständlich von einer Begrenzung des Notwehrrechts durch Verhältnismäßigkeitserwägungen ausgeht. ${ }^{69}$ Gerade die Tötung des Angreifers zur Verteidigung von Sachwerten wird mehrheitlich als nicht von $\mathbb{} 32$ StGB gedeckt angesehen - hier scheint also, um die Wendung von Popitz in einem anderen Kontext zu gebrauchen, eine Art „Präventivwirkung des Nichtwissens" ${ }^{70}$ zu greifen, die dem von Kritikern des schneidigen Notwehrrechts schon früh vorgebrachten Vorwurf der Förderung einer „Totschlags-

66 Wobei die Bereitschaft der Bevölkerung, Notwehr bzw. Nothilfe zu üben, je nach Art und Schwere des Angriffs sehr unterschiedlich und tendenziell eher gering ausgeprägt sein dürfte, vgl. die Nachweise bei

J. Renzikowski, Notwehr und Notstand, Berlin 1994, 80.

67 So auch K. Bernsmann ZStW 104 (1992), 290, 318 im Hinblick auf die mögliche Bewaffnung des Verteidigers.

68 S. bereits F. C. Schroeder, Die Notwehr als Indikator politischer Grundanschauungen, in: ders. (Hrsg.), Festschrift für Maurach, Heidelberg 1972, 127, 140; H. Koriath, FS Müller-Dietz (Fn. 38), 361.

69 K. Amelung/I. Kilian, Zur Akzeptanz des deutschen Notwehrrechts in der Bevölkerung, in: K. Amelung, Festschrift für Schreiber, Heidelberg 2003, 3 ff.; s. auch I. Kilian, Die Dresdener Notwehrstudie, Baden-Baden 2011, 39 ff. sowie dazu die Rezension von J. Kaspar Neue Kriminalpolitik 2012, 162. Auch A. Engländer, Grund und Grenzen der Nothilfe (Fn. 4), 16 weist auf diesen Umstand im Rahmen seiner Erörterung einer generalpräventiven Wirkung der Notwehr hin.

70 S. H. Popitz, Über die Präventivwirkung des Nichtwissens, Tübingen 1968. 
moral“ innerhalb der Bevölkerung ${ }^{71}$ entgegenwirkt. Würde man dagegen auf einen plakativen Abschreckungseffekt gerade durch das geltende Notwehrrecht setzen wollen, müsste man konsequenterweise Aufklärungskampagnen starten und diese Fehlvorstellung korrigieren. Ob man aber wirklich Werbetafeln mit Slogans wie „Wehr Dich auch mit Waffen - es ist Dein gutes Recht!“ sehen möchte, mag jeder selbst entscheiden.

Die Ergebnisse der Dresdener Notwehrstudie lassen sich auch als starkes Argument gegen eine positiv-generalpräventive Begründung des schneidigen Notwehrrechts heranziehen. Der in seinem Gehalt etwas schillernde und unbestimmte Aspekt der „Wiederherstellung des Rechtsfriedens“ soll bei der Strafe garantieren, dass diese von der Bevölkerung als gerechte und angemessene Reaktion auf die Straftat wahrgenommen werden kann, ohne, dass man diese Wirkung im Einzelfall ernsthaft empirisch messen könnte. Vielmehr obliegt es hier dem Gesetzgeber, durch seine Vorgaben normativ zum Ausdruck zu bringen, was er als gerechte und angemessene Reaktion ansieht - aber immer mit dem Ziel im Hinterkopf, damit den Vorstellungen der Gesellschaft entgegen zu kommen. Gibt es nun Anhaltspunkte dafür, dass die Bevölkerung auch mit einem niedrigeren Sanktionsniveau zufrieden ist bzw. einen völligen Verzicht auf eine Sanktionierung für akzeptabel erachtet, muss dem aus Gründen der Optimierung von Grundrechtsschutz nachgegangen werden; denn ansonsten liefe man Gefahr, eine Strafe zu verhängen, obwohl damit kein sinnvoller präventiver Effekt verbunden ist. ${ }^{72}$

Übertragen auf die Notwehr bedeutet das, dass ein besonders hartes Zurückschlagen des Angriffs aus Gründen der „Rechtsbewährung“ nicht legitimierbar ist, wenn die Bevölkerung eine solche Reaktion in bestimmten Konstellationen gar nicht erwartet, sondern im Gegenteil als übermäßig und rechtswidrig ansieht. Auch der Regierungsentwurf von 1962 geht interessanterweise davon aus, dass das Notwehrrecht in den Fällen eingeschränkt sei, ,in denen seine Ausübung von der Rechtsüberzeugung der Allgemeinheit missbilligt würde“73 - genau dies scheint bei der Tötung zur Verteidigung von Sachwerten aber eben der Fall zu sein. Demgegenüber ist die im selben Entwurf enthaltene Behauptung, wonach die Notwehr ein „schneidiges und seit alters her in der Rechtsüberzeugung des Volkes verwurzeltes Schutzrecht" sei, ${ }^{74}$ in dieser Allgemeinheit offensichtlich veraltet - vergleichbar der an ein antiquiertes bürgerliches Ehrverständnis anknüpfenden Behauptung, wonach dem Angegriffenen

71 A. Geyer in: F. von Holtzendorff (Hrsg.), Handbuch des deutschen Strafrechts, Bd. 4, Berlin 1877, 94, zitiert nach H. J. Hirsch, Die Notwehrvoraussetzung der Rechtswidrigkeit des Angriffs, in: H. H. Jescheck (Hrsg.), Festschrift für Dreher, Berlin 1977, 214.

72 S. dazu näher J. Kaspar, Verhältnismäßigkeit und Grundrechtsschutz im Präventionsstrafrecht (erscheint 2013).

73 BT-Drucks. IV/7650, 157.

74 BT-Drucks. IV/7650, 157. 
ein Ausweichen prinzipiell nicht zumutbar sei, weil dies eine „schimpfliche Flucht“ bedeuten würde. ${ }^{75}$ Denn auch dies findet in der Bevölkerung nach der genannten Studie keinen Widerhall. ${ }^{76}$ Insgesamt handelt es sich hier um eine der gar nicht so seltenen, bislang aber kaum übergreifend und systematisch erfassten Schnittstellen von Kriminologie und materiellem Strafrecht, bei denen es sich geradezu aufdrängt, empirische Erkenntnisse in die Auslegung und Anwendung von materiellen Strafgesetzen einfließen zu lassen. ${ }^{77}$

\section{Verfassungsrechtliche Konsequenzen}

Noch aus einem weiteren Grund lässt sich bei Annahme eines generalpräventiven Verständnisses von Rechtsbewährung die „Schneidigkeit“ des Notwehrrechts nicht begründen. Das wird deutlich, wenn man die oben bereits angedeutete verfassungsrechtliche Perspektive einblendet.

Dass zumindest die Tötung des Angreifers zur Verteidigung von Sachwerten ein verfassungsrechtliches bzw. menschenrechtliches Problem darstellt, hat Bernsmann bereits vor einiger Zeit mit guten Argumenten dargelegt ${ }^{78}$, auch wenn er mit seinen Thesen bisher kaum Gefolgschaft gefunden hat ${ }^{79}$. Für Bernsmanns Ansicht spricht zunächst die Wertung von Art. 2 II lit. a) EMRK, der gezielte Tötungen bei rechtswidrigen Angriffen nur zur „Verteidigung eines Menschen gegenüber rechtswidriger Gewaltanwendung“ erlaubt. Die EMRK richtet sich zwar unmittelbar nur an den Staat und gilt nicht für das Handeln von Privatpersonen. Sie gebietet aber eine menschenrechtskonforme Auslegung des einfachen Rechts, zu dem $\mathbb{3 2}$ StGB zählt ${ }^{80}$. Hinzu kommen Gesichtspunkte aus dem nationalen Verfassungsrecht. Aus Art. 2 II GG wird eine staatliche Pflicht zum Schutz des menschlichen Lebens abgeleitet ${ }^{81}$. Diesen Schutz „verwirkt“ der Angreifer auch nicht allein durch sein rechtswidriges

75 Vgl. (kritisch) E. Schmidhäuser, FS Honig (Fn. 18), 187: Es werde Sorge getragen, dass das ,altdeutschmannhaft-empfindsame Ehrgefühl“ keinen Schaden nehme. Zu Recht gegen entsprechende, noch drastischere Formulierungen bei Berner (man dürfe dem Volke nicht das „Gefühl seiner Männerwürde“ rauben) und $v$. Ihering („Abgrund von Verkommenheit des Persönlichkeitsgefühls, von Unmännlichkeit, von gänzlicher Entartung und Abgestumpftheit des einfachen, gesunden Rechtsgefühls“) $H$. Koriath, FS Müller-Dietz (Fn. 38)361, 384. Kritisch zum Gedanken der „schimpflichen Flucht“ auch C. Roxin, Strafrecht Allgemeiner Teil (Fn. 4), $\$ 15$ Rn. 50 sowie J. Bülte GA 2011, 156 (mit Beispielen aus der Rechtsprechung).

76 I. Kilian, Die Dresdener Notwehrstudie (Fn. 70), 77.

77 S. dazu J. Kaspar, Neue Kriminalpolitik 2012, $163 \mathrm{f}$.

78 K. Bernsmann ZStW 104 (1992), 290.

79 Ablehnend etwa C. Roxin, Strafrecht Allgemeiner Teil (Fn. 4), 15 Rn. 48.

80 Im Ergebnis ähnlich C. Roxin, Strafrecht Allgemeiner Teil (Fn. 4), $\mathbb{S} 15$ Rn. 87, der eine ,'Ausstrahlungswirkung' oder analoge Anwendung “ der EMRK im Hinblick auf das Notwehrrecht befürwortet. Begründet wird das interessanterweise auch mit dem Hinweis auf Rechtsbewährung als „öffentliche Funktion“ des Notwehrrechts, die „denselben Einschränkung wie die öffentliche Gewalt unterworfen werden müsse" (Roxin a.a.O.) - ein überzeugender Gedanke, der sich nach hier vertretener Ansicht aber nicht auf die Geltung der EMRK beschränken lässt, sondern auch die Geltung des (nationalen) Verfassungsrechts nahe legt. S. dazu sogleich oben im Text.

81 BVerfGE 39, 1, 42; aus jüngerer Zeit BVerfGE 115, 118, 152 (Luftsicherheitsgesetz). 
Verhalten $^{82}$, so dass die staatlich vorgesehene „Rechtmäßigkeit“ seiner Tötung auch in Fällen, wo nicht Leib, Leben oder sonstige gewichtige Rechtsgüter des Angegriffenen auf dem Spiel stehen, schon unter dem Aspekt der staatlichen Schutzpflicht bedenklich ist. Dabei wird nicht verkannt, dass dem Gesetzgeber bei der Erfüllung seiner Schutzpflicht vom BVerfG ein weiter Spielraum zugestanden und eine Verletzung dieser Pflicht nur im Ausnahmefall anerkannt wird ${ }^{83}$. Solange der Staat also irgendwelche Schutzmaßnahmen bereithält, die nicht gänzlich wirkungslos sind, genügt er regelmäßig den verfassungsrechtlichen Anforderungen. Hier liegen die Dinge jedoch insofern anders, als es nicht nur um bloßes staatliches Nichtstun gegenüber privaten Handlungen geht, die gegen das Leben eines Dritten (hier: des ursprünglichen Angreifers) gerichtet sind. Vielmehr erteilt der Staat mit $\mathbb{3} 32 \mathrm{StGB}$ dem angegriffenen Bürger schon vorab eine Genehmigung zu einem solchen Handeln - zugespitzt formuliert: eine „license to kill“ - und trägt damit selbst zur Herabsetzung des Schutzes des Lebens des Angreifers bei.

Nach hier vertretener Ansicht müsste man vor diesem Hintergrund und unter Einbeziehung der verfassungsrechtlichen Perspektive sogar noch einen Schritt weiter gehen, wenn man davon ausgeht, dass der Verteidiger bei seiner Angriffsabwehr zugleich zur generalpräventiv verstandenen „Rechtsbewährung“ beitragen soll. Denn dann spricht viel dafür, seine Handlung dem Staat zuzurechnen mit der Folge, dass sogar die Abwehrfunktion der Grundrechte unmittelbar aktiviert wird. Folgt man dem, wäre die von der h. M. bis heute nahezu einhellig vertretene sehr weitgehende „Suspendierung“ von Verhältnismäßigkeitserwägungen nicht mehr haltbar. ${ }^{84} \mathrm{Die}$ Begründung dieses revidierte Verständnis der Reichweite des Notwehrrechts kann hier nur angedeutet werden: „Generalprävention“ durch Maßnahmen zur Eindämmung von Straftaten zu betreiben ist ganz offensichtlich eine Aufgabe, die ansonsten

82 Das Gegenteil lässt sich auch der Entscheidung zum Luftsicherheitsgesetz (BVerfGE 115, 118) nicht entnehmen. Dort wird der Abschuss eines von Terroristen entführten Flugzeugs zwar aufgrund der eigenen Verantwortung der Handelnden nicht als Menschenwürdeverstoß qualifiziert (BVerfGE 115, 118, 161). Er wird sodann aber als schwerer Grundrechtseingriff (in das Grundrecht auf Leben) qualifiziert, der nur im Hinblick auf die damit bezweckte Rettung des Lebens unschuldiger Dritter - auch unter Berücksichtigung des Verhältnismäßigkeitsgrundsatzes - gerechtfertigt sei. Das betrifft zwar eine genuin abwehrrechtliche Konstellation, da allein staatliches Handeln im Raum stand, widerlegt aber zumindest das mögliche Argument einer „Verwirkung“ von Grundrechtsschutz durch schwere Straftaten.

83 BVerfGE 115, 118, 159.

84 A. A. die ganz h. M. Wie hier aber bereits J. Bülte GA 2011, 147, 159 f., der zudem (im Sinne der oben erwähnten Einordnung der Notwehrhandlung als strafähnliche Maßnahme völlig konsequent) die Wertung von Art. 102 I GG heranzieht. In der Tat läuft die Tötung, die zur Verteidigung individueller Güter nicht erforderlich, aber dennoch aus Gründen der „Rechtsbewährung“ zulässig sein soll, dem Gedanken des Art. 102 GG zuwider, wonach auch bei noch so schweren Straftaten eine Tötung (u. a.) aus generalpräventiven Gründen ausgeschlossen sein soll. Insofern ist es nicht unproblematisch, wenn das BVerfG in der Entscheidung zum Luftsicherheitsgesetz den Abschuss eines entführten Flugzeugs und die damit verbundene Tötung der „Straftäter“ (deren schuldhafte Tatbegehung zu diesem Zeitpunkt ja noch gar nicht feststeht!) damit legitimiert, dass diese für das von ihnen in Gang gesetzte Geschehen einschließlich zurechenbarer Folgen „,in Verantwortung genommen “ würden, vgl. BVerfGE $115,118,161 \mathrm{f}$. 
dem Staat vorbehalten ist. Wird der Verteidiger im Rahmen von $\$ 32$ StGB ermächtigt, diese Funktion mit zu übernehmen, verliert die Verteidigungshandlung ihren rein privaten Charakter und wird zugleich zur „quasi-staatlichen“ Handlung. Der Verteidiger agiert dann in einer Art Doppelrolle, bei der neben den Schutz der Individualinteressen das öffentliche Interesse an der Aufrechterhaltung der Rechtsordnung tritt. Zwar liegt keine förmliche Beleihung vor, aber, wie Freund zu Recht andeutet, eine ähnliche Konstellation, ${ }^{85}$ bei der dem Privaten eine Befugnis zur Erfüllung von an sich staatlichen Aufgaben erteilt wird. Nicht umsonst wird er als „Bewahrer und Repräsentant der Rechtsordnung “86 bezeichnet, dessen Tätigwerden „auch im öffentlichen Interesse“ ${ }^{87}$ liege.

Als Parallele kann man auf das private Festnahmerecht gem. \$127 I StPO verweisen, bei der nach Inhalt und systematischer Stellung der Regelung der „quasi-staatliche“ Charakter der Tätigkeit zwar noch deutlicher zu Tage tritt. Aber auch hier ist ja in aller Regel zugleich von einem privaten Nutzen der Festnahme eines auf frischer Tat Ertappten auszugehen. Warum man bei $\$ 127$ I StPO wie selbstverständlich von einer Geltung des Verhältnismäßigkeitsgrundsatzes bzw. des Übermaßverbots ausgeht, ${ }^{88}$ dies bei der insoweit ähnlich strukturierten Notwehr aber strikt ablehnt, erscheint nicht konsequent. In beiden Fällen handelt ein Privater (auch) zur Erfüllung staatlicher Aufgaben. Jedenfalls wenn man diese verfassungsrechtliche Überlegung mit einbezieht, ist die Kritik von Vertretern der Individualschutztheorie berechtigt, wonach schwer erklärlich ist, warum der bei originär staatlichem Handeln unstreitig zu beachtende Grundsatz der Verhältnismäßigkeit beim Handeln des Verteidigers keine Rolle spielen sollte. ${ }^{89}$ Dieser Ausgangspunkt nötigt allerdings nicht zwingend zur Aufgabe des Rechtsbewährungsgedankens; man kann ihn vielmehr, wie es hier vertreten wird, als ergänzendes Kriterium beibehalten und die damit verbundene gewisse zusätzliche Einschränkung des Notwehrrechts durch Verhältnismäßigkeitserwägungen befürworten. ${ }^{90}$

85 G. Freund, Strafrecht Allgemeiner Teil (Fn. 9), \$3 Rn. 86; vgl. auch J. Bülte GA 2011, 153: (, hoheitliche Aufgabe") sowie die bereits oben in Fn. 80 erwähnte ganz ähnliche Argumentation von $C$. Roxin, Strafrecht Allgemeiner Teil (Fn. 4), $\$ 15$ Rn. 87 im Hinblick auf die Geltung der EMRK.

86 T. Lenckner JZ 1973, 255.

87 H. Schröder JR 1962, 188.

88 Vgl. L. Meyer-Goßner, StPO, 55. Aufl., München2012, $\mathbb{1 2 7}$ Rn. 16.

89 Vgl. H. Frister GA 1988, 296 f.; A. Engländer, Grund und Grenzen der Nothilfe (Fn. 4), 26. Insofern ist ein „schneidiges“ Notwehrrecht bei monistisch-individualistischen Ansätzen sogar naheliegender, auch wenn natürlich auch hier der Aspekt der grundrechtlichen Schutzpflichten zu beachten wäre; dem kann hier nicht weiter nachgegangen werden.

90 Ablehnend A. Engländer, Grund und Grenzen der Nothilfe (Fn. 4), 90, der ausgehend von einem individualistischen und zugleich interessenbasierten Ansatz jegliche Einschränkung des Notwehrrechts über das Kriterium der Erforderlichkeit hinaus ablehnt; dass der Apfeldieb nicht vom Baum geschossen werden darf, wird dann lediglich extern über das sogenannte „Solidaritätsprinzip“ begründet (a.a.O., 355 ff.). Die Erwägung, dass es im Interesse des Angreifers (trotz seiner Verantwortung für die Angriffssituation!) begründbar ist, dass eine „extrem unverhältnismäßige“ Gegenwehr ausgeschlossen ist (a.a.O., 357 f.), lässt sich nach hier vertretener Ansicht aber durchaus auch auf die „einfach unverhältnismäßige“, insbesondere tödliche Gegenwehr übertragen. 


\section{E. Fazit: Restriktives Notwehrverständnis auf der Basis einer eingeschränkt- dualistischen Notwehrbegründung}

Als Fazit kann man also formulieren, dass gegen „Rechtsbewährung“ als Notwehrprinzip, wie es von vielen Vertretern einer dualistischen Auffassung verstanden und argumentativ verwendet wird, Bedenken bestehen. Es wird oft in zirkulärer Weise zur Begründung von Ausweitungen oder Einschränkungen des Notwehrrechts herangezogen, ohne dass zuvor Klarheit über die inhaltlichen Maßstäbe von „Rechtsbewährung“ geschaffen wird. Das zwingt aber nicht zur völligen Aufgabe des Prinzips. Dem Problem der inhaltlichen Unbestimmtheit von „Rechtsbewährung “ lässt sich, wie gezeigt, in gewissem Umfang dadurch begegnen, dass man auf eine Art generalpräventive Funktion der Notwehr hinweist. Sowohl empirisch-kriminologische als auch verfassungsrechtliche Erwägungen sprechen gegen die Annahme, dass sich die „Schneidigkeit“ des Notwehrrechts, insbesondere der Verzicht auf die Verhältnismäßigkeit der Gegenwehr, gerade mit einer solchen generalpräventiven Funktion erklären lässt. Paradoxerweise führt also ausgerechnet der Aspekt, der von der herrschenden Ansicht als Argument gegen eine Geltung von Verhältnismäßigkeitserwägungen herangezogen wird, zu einer Zurechnung des Verteidigungshandelns gegenüber dem Staat, was eine Bindung an die Grundrechte und damit den Verhältnismäßigkeitsgrundsatz mit sich bringt. Offensichtlich unverhältnismäßige Gegenwehr ist dem Angegriffenen auf dem Boden eines dualistischen Notwehrverständnisses daher nicht erlaubt.

Die zukünftige Aufgabe der Notwehrdogmatik wird es sein, die letztlich entscheidende notwehrspezifische Interessenabwägung genauer zu präzisieren und auszudifferenzieren. Dabei erscheint es zulässig, die Verantwortlichkeit des Angreifers einerseits $^{91}$ sowie die mögliche Überforderungssituation des Angegriffenen andererseits $^{92}$ ebenso in Rechnung zu stellen wie einen möglichen positiven Effekt der Verteidigung im Sinne negativer wie positiver Generalprävention. Etwas konkreter: Es darf in Rechnung gestellt werden, dass durch die Verteidigung nicht nur das konkret durch den Angriff gefährdete Rechtsgut geschützt wird, sondern zugleich ein Signal an potenzielle Angreifer gesendet wird, dass auch in Zukunft mit Gegenwehr zu rechnen sein wird. Auch das kann sich dann (aber eben wie gezeigt nur in gewissem Umfang und nur im Rahmen der Verhältnismäßigkeit) notwehrerweiternd auswirken. Vorzugswürdig ist also eine integrative Betrachtungsweise, bei der der Schutz individueller Güter die unverzichtbare Basis des Notwehrrechts bleibt und der Aspekt generalpräventiver Rechtsbewährung nur ergänzende Bedeutung im Rahmen der ohnehin vorzunehmenden Interessenabwägung hat. Er behält damit eine, wenn

91 S. J. Bülte GA 2011, 158.

92 Dazu K. Bernsmann ZStW 104 (1992), 290, 327; zustimmend H. Koriath, FS Müller-Dietz (Fn. 38) 361. 381, die zu Recht auf die Möglichkeit einer Entlastung des Verteidigers über eine (ggf. großzügiger als bislang zu handhabende) Exkulpation gem. $\$ 33 \mathrm{StGB}$ hinweisen. 
auch deutlich untergeordnete, Bedeutung; man könnte also von einer asymmetrischen, nur eingeschränkt dualistischen Theorie sprechen. ${ }^{93}$

\section{F. Zur Notwendigkeit einer Neuregelung}

$\mathrm{Ob}$ sich ein solches Notwehrverständnis unter Einschluss einer Interessenabwägung mit der lex lata verträgt, ist eine andere Frage. Zwar enthält $\ 32$ StGB im Unterschied zu den Notstandsregelungen keine explizite Abwägungsklausel. Es wäre aber zumindest denkbar, das Merkmal der "Gebotenheit“, an das schon bisher nach h. M. die sog. „sozial-ethischen Beschränkungen“ der Notwehr geknüpft werden, ${ }^{94}$ auch für eine solche etwas weitergehende Notwehreinschränkung heranzuziehen. ${ }^{95}$ Der Gesetzgeber des Jahres 1975 hat das Element der „Gebotenheit“ entgegen dem Entwurf von 1962 bewusst nicht gestrichen, sondern als Einbruchsstelle für Grenzziehungen durch die Rechtsprechung vorgesehen; ${ }^{96}$ dass er dabei den damaligen Rechtszustand zementieren wollte, lässt sich der Gesetzesbegründung nicht entnehmen.

Vorzugswürdig und der Rechtssicherheit dienlich wäre dennoch eine ausdrückliche Regelung, die man mit folgendem Wortlaut in einem neu zu schaffenden $\mathbb{} 32$ III platzieren könnte: ${ }^{97}$

„Die Notwehr ist ausgeschlossen, wenn sie offensichtlich außer Verhältnis zu dem durch den rechtswidrigen Angriff drohenden Schaden steht".

Als „Schaden“ ist dabei - dem eben skizzierten eingeschränkt-dualistischen Notwehrverständnis entsprechend - nicht nur die unmittelbar drohende Rechtsgutsverletzung angesprochen, sondern auch der Nebenaspekt einer möglichen generalpräventiven Einbuße bei Versagung des Notwehrrechts, der „Normgeltungsschaden“,

93 Im Ansatz ähnlich auch C. Roxin ZStW 93 (1981), 76, aber mit größerer Bedeutung der Rechtsbewährungskomponente. Mit der hier vorgenommenen (wenn auch nur skizzenhaften) Beschreibung der Funktion der beiden Prinzipien sowie ihres Verhältnisses zueinander wird dem (berechtigten) Vorwurf von

A. Engländer, Grund und Grenzen der Nothilfe (Fn. 4), 29, Rechnung getragen, wonach genau diese Frage bei Vertretern eines dualistischen Ansatzes oft zu kurz kommt.

94 Vgl. C. Roxin, Strafrecht Allgemeiner Teil (Fn. 4), $\$ 15$ Rn. 56; so auch G. Freund, Strafrecht Allgemeiner Teil (Fn. 9), $\$ 3$ Rn. 126; a. A. E. Schmidhäuser, FS Honig (Fn. 18), 194.

95 Vgl. V. Krey JZ 1979, 714, der ebenfalls (bereits de lege lata) von der Möglichkeit eines restriktiveren Verständnisses von $\$ 32$ StGB ausgeht. A. A. (und für eine Regelung de lege lata) aber J. Bülte GA 2011, $161 \mathrm{f}$.

96 BT-Drucks. V/4095, 14. So auch SSW-Rosenau, $\$ 32$ Rn. 31.

97 S. auch den Vorschlag der Kodifikation einer europäischen Notwehrregelung bei G. Dannecker, Der Allgemeine Teil eines europäischen Strafrechts als Herausforderung für die Strafrechtswissenschaft, in: T. Weigend /G. Küpper (Hrsg.), Festschrift für Hirsch, Berlin 1999, 141, 159, in der die Gefährlichkeit des Angriffs, die Schuld des Angreifers und der Wert des angegriffenen Rechtsguts als Abwägungsfaktoren genannt werden. Zustimmend Bülte GA 2011, 165. Die hier vorgeschlagene Neuregelung bezieht sich ausdrücklich nur auf das Problem der „unverhältnismäßigen“ Gegenwehr, auch wenn eine umfassende Kodifikation auch weiterer „sozialethischer Einschränkungen“ wünschenswert wäre. Dem kann hier nicht weiter nachgegangen werden. 
wenn man so will. ${ }^{98}$ Auch bei Berücksichtigung dieser potenziell notwehrerweiternden Wirkung des Rechtsbewährungsprinzips wäre das Notwehrrecht auf diese Weise dennoch seiner radikalen „Schneidigkeit“ beraubt. Es würde damit den nationalen verfassungsrechtlichen Vorgaben, den Notwehrregelungen fast aller anderen Länder, ${ }^{99}$ den Regelungen auf europäischer und internationaler Ebene sowie nicht zuletzt auch den Rechts- und Gerechtigkeitsvorstellungen eines ganz überwiegenden Teils der Bevölkerung deutlich besser entsprechen - eine in dieser Eindeutigkeit seltene Häufung von guten Gründen also, über eine gesetzliche Neuregelung ernsthaft nachzudenken. 